\title{
Cognitive Impairment and Associated Factors Among Adult Hypothyroid Patients in Referral Hospitals, Amhara Region, Ethiopia: Multicenter Cross-Sectional Study
}

This article was published in the following Dove Press journal:

Neuropsychiatric Disease and Treatment

\author{
Bezawit Mulat ${ }^{\prime}$ \\ Adugnaw Ambelu' \\ Sewbesew Yitayih ${ }^{2}$ \\ Yibeltal Yismaw Gela' \\ Ayechew Adera' \\ Yigizie Yeshaw (D)' \\ Yonas Akalu (D) \\ 'Department of Human Physiology, \\ School of Medicine, College of Medicine \\ and Health Sciences, University of \\ Gondar, Gondar, Ethiopia; ${ }^{2}$ Department \\ of Psychiatry, School of Medicine, College \\ of Medicine and Health Sciences, \\ University of Gondar, Gondar, Ethiopia
}

Correspondence: Bezawit Mulat Email mulatbezawit@gmail.com
Background: Cognitive impairment is a common complication of hypothyroidism that affects the patient's quality of life in different aspects. In Ethiopia, though the prevalence of hypothyroidism is high the magnitude of cognitive impairment among these patients is not known. Therefore, the current study is aimed at determining the prevalence of cognitive impairment and associated factors among adult hypothyroid patients.

Methods: An institution-based cross-sectional study was employed from February 24 to May 22, 2020, to assess the prevalence of cognitive impairment and associated factors among adult hypothyroid patients. Data was collected by a pretested intervieweradministered structured questionnaire and standardized Mini-mental state examination (SMMSE) tool. A cluster sampling technique was used. Data was entered into Epidata version 4.6 and exported into a statistical package for social science (SPSS) version 25 for further analysis. Both bivariable and multivariable logistic regression analyses were done. A 95\% confidence interval and P-value $<0.05$ were used to declare statistical significance.

Results: A total of 216 adult hypothyroid patients were included with a response rate of $98.6 \%$. The mean age was $42.5( \pm 11)$ years. The prevalence of cognitive impairment was $27.3 \%(95 \%$ CI $(21.7,33.8))$ and it was significantly associated with increased age (AOR = $1.23,95 \% \mathrm{CI}(1.11,1.34))$, increased duration of illness $(\mathrm{AOR}=1.48,95 \% \mathrm{CI}(1.14,1.90))$ and high thyroid-stimulating hormone (TSH) level (AOR $=1.3,95 \%$ CI $(1.1,1.6)$ ).

Conclusion: The prevalence of cognitive impairment among hypothyroid patients was high. Increased age, increased duration of illness and high TSH levels were significantly associated with cognitive impairment. Hence, early screening of cognitive impairment among hypothyroid patients with increased age, increased duration of illness and high TSH level should be given due emphasis to increase patients quality of life.

Keywords: cognitive impairment, hypothyroidism, SMMSE, Ethiopia

\section{Introduction}

Hypothyroidism or underactive thyroid is a failure of the thyroid gland to produce enough thyroid hormone to meet the metabolic demands of the body. It may occur as a result of primary gland failure or insufficient thyroid gland stimulation by the hypothalamus and anterior pituitary gland. ${ }^{1}$

Cognitive impairment is one of the common complications that occur among patients with hypothyroidism. ${ }^{2}$ It is a condition in which a person has suffered in recalling, learning new things, concentrating, and making decisions that affect his/ 
her everyday life. ${ }^{3}$ When cognitive impairment occurs it can affect a range of cognitive domains including attention, concentration, language, memory, psychomotor and executive functions. ${ }^{4}$

There are different causes for cognitive impairment such as genetic factors, exposure to toxic substances, head injury, chronic illnesses, malnutrition, and other lifestyle factors like smoking, chat chewing, and alcoholism. From those wide ranges of causes, hypothyroidism is one of the diseases of the thyroid gland that can cause cognitive impairment. ${ }^{5}$ Hypothyroidism prevents the brain from adequately sustaining the energy (glucose) needed for neurotransmission, memory, and other higher brain functions and it also causes biochemical and biophysical changes in the hippocampus which is important for cognition. ${ }^{6}$

Even though there is no global data on to what extent cognitive impairment affects patients with hypothyroidism, there are pieces of evidence across different parts of the world that claimed cognitive impairment is a common problem among these patients. For instance, cognitive impairment affected about $17 \%, 28 \%$, and $32 \%$ of hypothyroid patients in Minnesota, Los Angeles, and India respectively. ${ }^{7-9}$

Cognitive impairment affects an individual's functional ability especially instrumental activities of daily life (IADL), such as the use of the telephone, transportation, management of their medications, and money. ${ }^{10}$ People with cognitive impairment use their senses (sight, hearing, and touch) as a compensatory mechanism for declining cognition. It is reported that they use these strategies to gain a sense of control over their lives rather than to be as effective as before. ${ }^{11}$ Sensory processing refers to the ability to register and modulate sensory information and to organize this sensory input to respond to situational demand. ${ }^{12,13}$ Patients with major depressive disorder (MDD) also experienced cognitive deficits, such as visual and verbal memory, working memory, attention, executive function, and processing speed, ${ }^{14}$ but the most significant impairment is appreciated in executive function. ${ }^{15,16}$ A defect in cognitive control, which refers to the ability to regulate one's thoughts and actions to achieve internal goals, has been associated with depression-related complications including suicidality. ${ }^{17,18}$

Above all low thyroid hormone levels have increased risk of occurrence of dementia commonly among aged females unless the hormone level is corrected early. ${ }^{19}$
Even though there are no population-based studies to assess the prevalence of hypothyroidism some institutionbased studies revealed that hypothyroid disorder is high in Ethiopia. $^{20,21}$ Despite, hypothyroidism is prevalent and one of the endocrine disorders that can lead to cognitive impairment, there is a paucity of data regarding the prevalence of cognitive impairment and factors associated with it among adult patients with Hypothyroidism. Therefore, this study aimed to assess the prevalence of cognitive impairment and associated factors among adult hypothyroid patients.

\section{Methods and Materials Study Area and Period}

An institution-based cross-sectional study was employed at Amhara Regional State selected Referral Hospitals from February 24 to May 22, 2020, to assess the prevalence of cognitive impairment and associated factors among adult hypothyroid patients. The selected referral hospitals were the University of Gondar Specialized and Comprehensive, Felege Hiwot, and Debre Markos referral hospitals.

\section{Criteria for Inclusion and Exclusion}

All already diagnosed adult hypothyroid patients who are on appropriate levothyroxine replacement therapy were included in the study.

Patients who had a history of seizure, epilepsy, stroke, critically ill, had a hearing problem and had known severe psychiatric illness (schizophrenia, major depressive disorder (MDD), bipolar disorders, obsessive-compulsive disorder (OCD), and dementia were excluded.

\section{Sample Size Determination and Sampling Technique}

The actual sample size was determined by using the single population proportion formula. Since there was no similar study conducted in Ethiopia, the following assumptions have been made: $95 \%$ confidence interval $(Z \alpha / 2=1.96)$, $50 \%$ proportion, and $5 \%$ margin of error.

$$
n=\frac{(\mathrm{Za} 2)^{2} p(q)}{d^{2}} \text {, where } \mathrm{Z} \text { statistics (at } 95 \% \mathrm{CI}=1.96 \text { ), }
$$
$\mathrm{p}$ (population proportion), and $\mathrm{d}$ (margin of error)

$$
n=\frac{(1.96)^{2} \times 0.5 \times 0.5}{0.05^{2}}=384
$$

However, based on the six hospitals' monthly report of hypothyroid cases, the total number of patients who could present during the data collection period was 460 which 
was $<10,000$. Hence we had used a population correction formula to determine our final sample size.

Population correction formula, $n f=\frac{n}{1+\frac{n}{N}}$ Where:

- $\mathrm{nf}=$ final sample size

- $\mathrm{n}=$ sample size before population correction

- $\mathrm{N}=$ total number of patients who could be presented

$$
n f=\frac{384}{1+\frac{384}{460}}=209
$$

Finally, we had added a 5\% non-response rate which made our final sample size 219.

A single-stage cluster sampling technique was employed to recruit the study participants. In the first step, six referral hospitals found in Amhara Regional State (University of Gondar Comprehensive and Specialized Referral Hospital (UOGCSH), Felege Hiwot Referral Hospital (FHRH), Debre Markos Referral Hospital (DMRH), Tibebe Gion Referral Hospital (TGRH), Dessie Referral Hospital (DRH), and Debre Berhan Referral Hospital (DBRH) were considered as a cluster and three of them (University of Gondar Comprehensive Specialized Referral Hospital (UOGCSH), Felege Hiwot Referral Hospital (FHRH) and Debre Markos Referral Hospital (DMRH)) were selected on a random basis. Following that, we included all patients who had to follow up at the outpatient department (OPD) and those who were receiving inpatient services on the selected referral hospitals.

\section{Study Variables \\ Dependent Variable \\ Cognitive impairment}

\section{Independent Variables}

Socio-demographic variables (including age, sex, marital status, educational status, and job status), comorbid medical conditions (including hypertension, cardiovascular diseases, diabetes mellitus, and chronic kidney disease), behavioral factors like khat chewing, alcohol drinking, and cigarette smoking, thyroid function tests (TSH, T4 \& T3 levels) and duration of illness.

\section{Operational Definition Hypothyroidism}

Serum TSH level $>4.5 \mathrm{mIU} / \mathrm{L}, \mathrm{FT} 4<10.3 \mathrm{pmol} / \mathrm{I}$ or FT3 $<2.3 \mathrm{pmol} /$ diagnosed as hypothyroid and were included in the study. ${ }^{20,22}$

\section{Data Collection Tools and Procedures}

Data was collected by a pretested interviewer-administered structured questionnaire and SMMSE tool. The questionnaire was prepared by the investigator after reviewing different pieces of literature..$^{7-9,23,24}$ The recent TSH and thyroid hormone levels were taken from the patient's medical chart. Cognitive impairment was assessed through a face to face interviews by using the SMMSE tool used by other studies conducted in Ethiopia among type 2 diabetic patients. ${ }^{25}$ The SMMSE has five components including orientation, registration, attention, and calculation, recall and language each having 10,3, 5, 3, and 9 items respectively. ${ }^{26}$ For participants who cannot read and write, the last three items measuring the language component of SMMSE were excluded since they require writing or reading skills. ${ }^{27}$ Participants who had SMMSE score below 22 with no or primary level education, less than 24 for those with secondary level education, and less than 25 for those with diploma and above educational status were diagnosed to have cognitive impairment. ${ }^{25}$

The data collectors used hand sanitizers, gloves, and face masks throughout the data collection process following the introduction of the coronavirus pandemic. In addition to this, the face-to-face interview was done by keeping the recommended distance ( 2 meters) to prevent coronavirus transmission. Moreover, each participant was asked for the symptoms of the coronavirus infection by the data collectors before starting any data collection in addition to the screening service given by the hospitals.

\section{Data Quality Assurance}

Initially, the questionnaire was developed in English by the investigator then forward and backward translation was done by Amharic and English versed personals to keep its consistency. Necessary amendments were made to the questionnaire based on the translation. A one-day training was given to both the data collectors and supervisors by the principal investigator about the purpose of the study, data collection procedures, and ethical issues during data collection.

A pretest was done at Tibebe Gion Referral hospital among 12 hypothyroid patients $(5 \%$ of the total sample size) for the assessment of the questionnaire clarity and sociocultural compatibility. Moreover, cognitive impairment was assessed by using a commonly used screening tool (SMMSE) with sensitivity and specificity of $84.1 \%$ and $73 \%$ respectively. ${ }^{28}$ In addition to this, the reliability 
of the instrument was established based on the internal consistency reliability assessment using Cronbach alpha $(\alpha=0.84){ }^{29}$

\section{Data Processing and Analysis}

The collected data were checked for completeness, accuracy, and clarity before analysis. The data were entered into Epidata 4.6 and transferred to SPSS version 25 for analysis. Descriptive statistics, frequencies, and proportions were computed. The total score of the SMMSE was used to screen cognitive impairment. Bivariable binary logistic regression analysis was employed to identify factors that are candidates for multivariable analysis at a p-value of less than 0.2. Multivariable binary logistic regression analysis was done to declare the presence of statistical significance. Model fitness was checked with the Hosmer and Lemeshow goodness of fit test and it was 0.1 . Furthermore, multicollinearity between the explanatory factors was assessed with the variance inflation factor (VIF) to identify and avoid redundant variables that may affect our estimate. The VIF was in the acceptable range (1-4). Indeed, a p-value $<0.05$ and a $95 \%$ confidence interval was used to determine statistical significance.

\section{Results}

A total of 216 hypothyroid patients were included in the study with a response rate of $98.6 \%$. The mean age of the participants was $42.5( \pm 11)$ years with an age range of 18-72 years. The majority $(73.1 \%)$ of the participants were females. (Table 1).

\section{Clinical Characteristics and Substance Use History of the Participants}

Of the total respondents, $13(6 \%)$ of them had known comorbid illness. Specifically, 5 (2.3\%) of them were hypertensive, $4(1.9 \%)$ had heart disease and $4(1.9 \%)$ of them had other comorbidities (gastritis, asthma). The mean duration of the illness (hypothyroidism) was $4.5( \pm 2.6)$ years and ranges from 1-13 years. The mean TSH level among them was $6.98 \pm 6.8(\mathrm{mIU} / \mathrm{L})$.

Regarding substance usage, more than half (53.7\%) of the study participants had a lifetime history of alcohol intake. Of those who had a lifetime alcohol history, 65 $(56 \%)$ of them had a history of alcohol intake history in the last one month. All most of them (98\%) were used to drink local beverages, tella, or areki. Indeed, only $9(4.2 \%)$
Table I Sociodemographic Characteristics of Adult Hypothyroid Patients Who Attend Amhara Regional State Referral Hospitals, Amhara Ethiopia $2020(n=216)$

\begin{tabular}{|l|l|l|l|}
\hline \multicolumn{2}{|l|}{ Variable Category } & Frequency & Percent \\
\hline Age (in years) & $18-31$ & 37 & 17.1 \\
& $32-45$ & 93 & 43.0 \\
& $46-59$ & 66 & 30.6 \\
& $\geq 60$ & 20 & 9.3 \\
\hline Sex & Male & 58 & 26.9 \\
& Female & 158 & 73.1 \\
\hline Marital status & Single & 51 & 23.6 \\
& Married & 140 & 64.8 \\
& Widowed & 19 & 8.8 \\
& Other* & 6 & 2.8 \\
\hline Residence & Rural & 145 & 67.1 \\
& Urban & 71 & 32.9 \\
\hline Educational status & Grade 8 and lower & 180 & 83.3 \\
& Grade 9-12 & 18 & 8.3 \\
& Diploma and above & 18 & 8.4 \\
\hline Monthly income & $\leq 585$ & 143 & 66.2 \\
(ETB) & $586-1650$ & 28 & 13.0 \\
& $1651-3145$ & 27 & 12.5 \\
& $\geq 3146$ & 18 & 8.3 \\
\hline Job-status & Employed & 133 & 61.6 \\
& Unemployed & 83 & 38.4 \\
\hline
\end{tabular}

Note: *Divorced.

Abbreviation: ETB, Ethiopian birr.

and $1(0.5 \%)$ of the study participants had ever Khat chewing and ever smoking history, respectively (Table 2).

We have recorded the frequency and amount of alcohol that the patients took but are difficult to state because most of the participants took local beverages and they are unable to recall the amount and the frequency of alcohol since they drink occasionally.

Alcohol abuse was assessed by masking symptoms of alcohol abuse such as drinking to relax, problems with family and friends because of drinking, neglecting their responsibilities because of drinking, and whether they were experiencing legal problems because of alcohol drinking) but there was no participant with such experiences.

\section{Prevalence of Cognitive Impairment}

Based on the adjusted SMMSE score the prevalence of cognitive impairment was $27.3 \%$ (95\% CI $(21.7,33.8)$ ). The prevalence of cognitive impairment was higher among females $50(31.6 \%)$ and among participants from rural 
Table 2 Clinical Characteristics and Substance Use History of Adult Hypothyroid Patients Who Attended Amhara Regional State Referral Hospitals, Amhara, Ethiopia, 2020 ( $n=216)$

\begin{tabular}{|l|l|l|l|}
\hline Variables & Category & Frequency & Percent \\
\hline Comorbid illness & Yes & 13 & 6 \\
& No & 203 & 94 \\
\hline Lifetime alcohol use & Yes & 116 & 53.7 \\
& No & 100 & 46.3 \\
\hline Other substance use & Yes & 10 & 4.6 \\
& No & 206 & 95.4 \\
\hline $\begin{array}{l}\text { Thyroid function } \\
\text { tests }\end{array}$ & TSH (mlU/L) & 6.98 & 6.86 \\
& T4 (pmol/L) & $9.6 *$ & $(8.2-12.2)^{* *}$ \\
\hline $\begin{array}{l}\text { Duration of illness } \\
\text { (years) }\end{array}$ & & 2.7 & 7.6 \\
\hline
\end{tabular}

Notes: Other substances (khat and cigarettes), *median, **interquartile range, $\mathrm{C}_{\text {continuous. }}$

areas $44(30.3 \%)$ as compared to their counterparts. Moreover, the prevalence of cognitive impairment was found to be higher among participants having an educational level of grade 8th and lower, 54 (30\%) than participants having secondary and above educational status. The mean TSH level of participants with cognitive impairment was $9.5( \pm 12.4) \mathrm{mIU} / \mathrm{L}$ and it was higher than participants with normal cognitive function.

\section{Factors Associated with Cognitive Impairment}

In the bivariable logistic regression analysis, independent variables with $\mathrm{p}$ - the value of less than 0.2 were passed to be included in the multivariable logistic regression analysis. Those variables were age, sex, marital status, residence, educational status, monthly income, duration of the illness, and TSH level. The output of the multivariable logistic regression analysis showed that age $(\mathrm{AOR}=1.23$, $95 \%$ CI $(1.11,1.34)$ ), duration of illness (AOR $=1.48$, $95 \% \mathrm{CI}(1.14,1.9))$, and $\mathrm{TSH}$ level $(\mathrm{AOR}=1.3,95 \% \mathrm{CI}$ $(1.1,1.6))$ of the participants were significantly associated with hypothyroid associated cognitive impairment. A unit increase in age of the participant increases the odds of having cognitive impairment by $23 \%$ and as the duration of illness (hypothyroidism) increases the odds of having cognitive impairment among the participants was increased by $48 \%$. Finally, a unit increase in the TSH level of the participants increases the odds of having cognitive impairment by $30 \%$ (Table 3 ).

\section{Discussion}

This study assessed the prevalence of cognitive impairment and associated factors among adult hypothyroid patients in Amhara regional state selected referral hospitals.

In the present study, the prevalence of cognitive impairment among adult hypothyroid patients was $27.3 \%$ (95\% CI $(21.7,33.8)$, and it was significantly associated with increased age, increased duration of illness, and high thyroid-stimulating hormone (TSH) level.

This result is in harmony with the study done in India $(32.2 \%)^{9}$ and Los Angeles $(28 \%){ }^{8}$ On the other hand, the result of the present study is higher than that of the study conducted in Minnesota $(17 \%) .^{7}$ The discrepancy may be elucidated by differences in sociodemographic characteristics of the participants which are educational level. Higher educational levels among Minnesota participants might be associated with health literacy that increases the degree to which individuals can obtain, process, and understand basic health information and services needed to make appropriate health decisions. ${ }^{30}$ In addition to the above, the discrepancy may be due to the use of different assessment techniques. The study conducted in Minnesota use extensive psychometric tests involved 9 cognitive tests (Logical Memory-II (delayed recall) and Visual Reproduction-II (delayed recall) from the Wechsler Memory Scale-Revised and the Auditory Verbal Learning Test for memory domains, Trail Making Test B, and Digit Symbol Substitution from Wechsler Adult Intelligence Scale-Revised for executive function; Boston Naming Test and Category Fluency Test for language; and Picture Completion and Block Design from the Wechsler Adult Intelligence Scale-Revised for visuospatial skills) and age-adjusted scaled scores in each domain were summed to obtain the domains score, and impairment in a domain was determined by comparing the scores with the mean (SD) of the population norm since the study assesses each domain in-depth it may decrease overestimation of cognitive impairment. ${ }^{7}$ Indeed it may decrease the prevalence of cognitive impairment among hypothyroid patients in Minnesota.

In the current study, cognitive impairment among adult hypothyroid patients was significantly associated with increased age, which is supported by a study conducted in Los Angeles. ${ }^{8}$ This increased prevalence of cognitive impairment with an increase in age could be due to aginginduced changes in T4 transport into the brain areas that are important for cognitive function, altered brain conversion of T4 into T3, decreased brain thyroid receptor number or 
Table 3 Factors Associated with Cognitive Impairment Among Adult Hypothyroid Patients in Bivariable and Multivariable Binary Logistic Regression, Amhara Regional State Referral Hospitals, Amhara, Ethiopia, 2020 ( $n=216$ )

\begin{tabular}{|c|c|c|c|c|}
\hline \multirow[t]{3}{*}{ Variable } & \multicolumn{2}{|c|}{ Cognitive Impairment } & \multirow[t]{3}{*}{ COR } & \multirow[t]{3}{*}{ AOR } \\
\hline & Yes $(n=59)$ & No $(n=\mid 57)$ & & \\
\hline & Frequency (\%) & Frequency (\%) & & \\
\hline Age (in years) ${ }^{c}$ & $53.7( \pm 8.5)$ & $38.3( \pm 8.7)$ & $1.29(1.19,1.38)$ & $1.23(1.11,1.34)^{*}$ \\
\hline \multicolumn{5}{|l|}{ Sex } \\
\hline Male & $9(15.5)$ & $49(84.5)$ & I & \\
\hline Female & $50(31.6)$ & $108(68.4)$ & $2.5(1.15,5.53)$ & $0.82(0.22,3.1)$ \\
\hline \multicolumn{5}{|l|}{ Marital status } \\
\hline Single & $3(5.9)$ & $48(94.1)$ & $0.04(0.01,0.17)$ & $0.37(0.04,3.27)$ \\
\hline Married & $4 \mid(29.3)$ & 99 (70.7) & $0.28(0.12,0.67)$ & $1.5(0.40,5.5)$ \\
\hline Others & $15(60))$ & $10(40)$ & I & \\
\hline \multicolumn{5}{|l|}{ Residence } \\
\hline Rural & $44(30.3)$ & 101 (69.6) & $1.6(0.83,3.18)$ & $0.54(0.12,2.3)$ \\
\hline Urban & $15(21.1)$ & $56(78.9)$ & I & \\
\hline \multicolumn{5}{|l|}{ Educational level } \\
\hline Grade 8 and lower & $54(30)$ & $126(70)$ & $2.66(0.98,7.2)$ & \\
\hline$\geq$ grade 9 & $5(13.9)$ & $31(86.1)$ & 1 & \\
\hline \multicolumn{5}{|l|}{ Job-status } \\
\hline Employed & $35(26.3)$ & $98(73.6)$ & I & \\
\hline Unemployed & $24(28.9)$ & $59(7 \mid .1)$ & $1.14(0.62,2.1)$ & \\
\hline Monthly income $(\mathrm{ETB})^{\mathrm{c}}$ & $635( \pm \mid 203)$ & $926( \pm 132)$ & I $(1.0,1.01)$ & I $(0.99,1.00)$ \\
\hline Duration of illness (in years) ${ }^{c}$ & $7.3( \pm 2.5)$ & $3.5( \pm 1.75)$ & $2.12(1.7,2.6)$ & $1.48(1.14,1.9)^{*}$ \\
\hline TSH level $(\mathrm{mlU} / \mathrm{L})^{c}$ & $9.5( \pm 12.4)$ & $6( \pm 2)$ & $1.4(1.17,1.67)$ & I.3 (I.I, I.6)* \\
\hline T4 level (pmol/L) $)^{c}$ & $9.0(7.3-10.7)^{\neq}$ & $10.1 \mid(8.2-12.2)^{\neq}$ & $0.9(0.99,1.01)$ & \\
\hline T3 level $(\mathrm{pmol} / \mathrm{L})^{\mathrm{c}}$ & $2.2( \pm 0.83)$ & $2.95( \pm 8.9)$ & $0.96(0.77,1.21)$ & \\
\hline
\end{tabular}

Notes: *Statistically significant, ${ }^{\neq}$median (interquartile range), ${ }^{C}$ continuous.

Abbreviations: $\mathrm{ETB}$, Ethiopian birr; $\mathrm{Cl}$, confidence interval; $\mathrm{COR}$, crude odds ratio; $\mathrm{AOR}$, adjusted odds ratio.

affinity. Decreased T4 and T3 levels might be associated with a decreased metabolic activity that consequently decreases the brain glucose-consuming process needed for neurotransmission, memory, and other higher brain functions involved in cognitive function. ${ }^{23}$ Naturally, the distribution of nuclear T3 receptors varies with the region and the cell types, and the highest concentration is found in the amygdala, hippocampus, and cortex. Hence the higher functions of the brain including cognition are dependent on the amount of these receptors in the neurons of the aforementioned areas of the Central Nervous System (CNS). ${ }^{31}$ Indeed the decrement of thyroid receptors among aged hypothyroid patients can increase the prevalence of cognitive impairment. Additionally, it may be due to age-related changes in the structure and function of synapses and changes in neuronal networks involved in higher brain functions including cognition. ${ }^{32}$ In fact, in advanced age, the cognitive capabilities of individuals also drop in a very impressive manner and make neuronal functions defective in processing and integrations of information. ${ }^{33}$

Longer duration of illness was another independent factor that was significantly associated with cognitive impairment among hypothyroid patients in the present study. This finding is supported by a study conducted in Canada. ${ }^{23}$ As the duration of thyroid hormone deficiency becomes longer it can cause severe damage in brain areas that had a significant role in cognitive functions, as a result, it might be accompanied by increased cognitive impairment. ${ }^{34}$ Furthermore, in the present study cognitive impairment among hypothyroid patients was significantly 
associated with increased TSH level. Which is supported by studies conducted in India, ${ }^{9}$ and in the united kingdom. ${ }^{35}$

\section{Limitation of the Study}

It may not possible to establish causal relationships because of the cross-sectional nature of the study. In addition to this, undiagnosed mental illnesses might have affected the performance of the study subjects on the SMMSE items, and then on the overall score. Moreover, the tool (SMMSE) used to assess cognitive impairment was not validated for the study population. Hence, the measurement results may over or underestimated the outcome of interest despite this the reliability of the instrument was in the acceptable range.

\section{Strength of the Study}

In the presence of the aforesaid limitations, the present study addresses the overlooked issue in hypothyroid patients and it could be consumed by successive researchers as preliminary information on the area of interest.

\section{Conclusion}

The prevalence of cognitive impairment among adult hypothyroid patients was high. Increased age, increased duration of illness and high TSH level were factors that were associated with cognitive impairment. Hence, early screening of cognitive impairment among hypothyroid patients with increased age, increased duration of illness and high TSH level should be given due emphasis to increase patients quality of life and to decrease economic burden on individuals, family, and country level. Moreover, early screening can decrease the progression of mild cognitive impairment to dementia by giving appropriate replacement therapy.

\section{Recommendation}

Periodic screening of cognitive functions among hypothyroid patients with increased age increased duration of illness, and high TSH levels to prevent further complications should be given due emphasis.

Longitudinal studies are good to assess causality and decrease errors made in the documentation of TSH and thyroid hormone levels.

\section{Abbreviations}

CI, Confidence Interval; FT4, Free Thyroxin; FT3, Free Triiodothyronine; IQR, Inter Quartile Range; SMMSE, Standardized Mini-Mental State Examination; SPSS,
Statistical Package for Social Science; TSH, Thyroid Stimulating Hormone.

\section{Data Sharing Statement}

All data are available in the manuscript and can be uploaded based on request.

\section{Ethical Approval and Consent to Participate}

Ethical clearance was obtained from the ethical review committee of the School of Medicine, College of Medicine and Health Sciences, University of Gondar. An official letter was submitted to the University of Gondar Comprehensive Specialized Hospital, Felege Hiwot Referral Hospital, and Debre Markos Referral Hospital and respective official permission was obtained. The study was conducted under the Declaration of Helsinki. Written informed consent was obtained from all study participants and confidentiality was kept. All the study participants answered the administered pre-tested questionnaires voluntarily and confidently. Furthermore, COVID-19 protective measures were used by data collectors and supervisors.

\section{Acknowledgments}

First, we would like to thank the University of Gondar, College of Medicine and Health Sciences, School of Medicine, Department of Physiology for giving us the chance to pursue the study.

Secondly, we are grateful to thank the University of Gondar Comprehensive and Specialized Referral Hospital, Felege Hiwot Referral Hospital, and Debre Markos Referral Hospital for their cooperation and permission were given to us during the data collection process. Finally, we want to pass our gratitude to study participants for their willingness and time to participate in the study. On top of this, we would like to thank data collectors for their commitment during the data collection period.

\section{Author Contributions}

All authors made substantial contributions during conception and design, acquisition of data, or analysis and interpretation of data; took part in drafting the article or revising it critically for important intellectual content; agreed to submit to the current journal; gave final approval of the version to be published; and agree to be accountable for all aspects of the work. 


\section{Disclosure}

The authors report no conflicts of interest for this work.

\section{References}

1. Anandkumar S, Venkatesh S. Clinical assessment of cognitive impairment in various conditions of hypothyroidism patients. World $J$ Pharm Res. 2019;8(7):1545-1555.

2. Smith CD, Grondin R, Lemaster W, Martin B, Gold BT, Ain KB. Reversible cognitive, motor, and driving impairments in severe hypothyroidism. Thyroid. 2015;25(1):28-36. doi:10.1089/thy.2014.0371

3. Bronas UG, Puzantian H, Hannan M. Cognitive impairment in chronic kidney disease: vascular milieu and the potential therapeutic role of exercise. Biomed Res Int. 2017;2017:1-10. doi:10.1155/2017/2726369

4. Samuels MH. Cognitive function in untreated hypothyroidism and hyperthyroidism. Curr Opin Endocrinol Diabetes Obes. 2008;15 (5):429-433. doi:10.1097/MED0b013e32830eb84c

5. Steckl C, Staats N. Various causes of cognitive disorders [Internet]; 2017. Available from: https://www.mentalhelp.net/cogni tive-disorders/various-causes/. Accessed February 10, 2020

6. Bauer M, Silverman DHS, Schlagenhauf F, et al. Brain glucose metabolism in hypothyroidism: a positron emission tomography study before and after thyroid hormone replacement therapy. $J$ Clin Endocrinol Metab. 2009;94(8):2922-2929. doi:10.1210/jc.2008-2235

7. Parsaik AK, Singh B, Roberts RO, et al. Hypothyroidism and risk of mild cognitive impairment in elderly persons a population-based study. JAMA Neurol. 2014;71(2):201-207. doi:10.1001/jamaneurol.2013.5402

8. Osterweil D, Syndulko K, Cohen SN, et al. Cognitive function in non-demented older adults with hypothyroidism. J Am Geriatr Soc. 1992;40(4):325-335. doi:10.1111/j.1532-5415.1992.tb02130.x

9. Bajaj S, Sachan S, Misra V, Varma A, Saxena P. Cognitive function in subclinical hypothyroidism in elderly. Indian J Endocrinol Metab. 2014;18(6):811. doi:10.4103/2230-8210.141355

10. Avlund K, Fromholt P. Instrumental activities of daily living: the relationships to self-rated memory and cognitive performance among 75-year-old men and women. Scand J Occup Ther. 1998;5 (2):93-100. doi:10.3109/11038129809035734

11. Nygård L, Öhman A. Managing changes in everyday occupations: The experience of persons with Alzheimer's disease. Occup Ther $J$ Res. 2002;22(2):70-81.

12. Humphry R. Young children's occupations: explicating the dynamics of developmental processes. Am J Occup Ther. 2002;56(2):171-179. doi:10.5014/ajot.56.2.171

13. Miller LJ, Anzalone ME, Lane SJ, Cermak SA, Osten ET, Lane SJ. Concept evolution in sensory integration: a proposed nosology for diagnosis. Am J Occup Ther. 2002;135-140.

14. Rock PL, Roiser JP, Riedel WJ, Blackwell AD. Cognitive impairment in depression: a systematic review and meta-analysis. Psychol Med. 2014;44(10):2029-2040. doi:10.1017/S0033291713002535

15. Austin M, Mitchell P, Goodwin M. Cognitive deficits in depression: possible implications for functional neuropathology Cognitive deficits in depression Possible implications for functional neuropathology. $\mathrm{Br}$ J Psychiatry. 2013;178(3):200-206. doi:10.1192/bjp.178.3.200

16. Hammar Å, Årdal G. Cognitive functioning in major depression - A summary. Front Hum Neurosci. 2009;3(SEP):1-7. doi:10.3389/ neuro.09.026.2009

17. Braver TS. The variable nature of cognitive control: a dual mechanisms framework. Trends Cogn Sci. 2012;16(2):106-113. doi:10.1016/ j.tics.2011.12.010
18. Serafini G, Pompili M, Innamorati M, Rihmer Z, Sher L, Girardi P. Can cannabis increase the suicide risk in psychosis? A critical review. Curr Pharm Des. 2012;18(32):5165-5187. doi:10.2174/138161212802884663

19. Chaker L, Bianco AC, Jonklaas J, Peeters RP. Hypothyroidism. Lancet. 2017;390(10101):1550-1562. doi:10.1016/S0140-6736(17) 30703-1

20. Reta Demissie W. Prevalence, clinical presentation and patterns of thyroid disorders among anterior neck mass patients visiting Jimma Medical Center, Southwest Ethiopia. Biomed J Sci Tech Res. 2019;18(2).

21. Kebede D, Abay Z, Feleke Y. Pattern, clinical presentations and management of thyroid diseases in national endocrine referral clinics, Tikur Anbessa Specialized Hospital, Addis Ababa, Ethiopia. Ethiop Med J. 2012;50(4):287-295.

22. Baskin HJ, Duick DS, Guttler RB, et al. American Association of Clinical Endocrinologists Medical Guidelines for Clinical Practice for the Evaluation and Treatment of American Association of Clinical Endocrinologists Medical Guidelines for Clinical Practice. AACE thyroid task force. Endocr Pr. 2002;8(6):457-469.

23. Bégin ME, Langlois MF, Lorrain D, Cunnane SC. Thyroid function and cognition during aging. Curr Gerontol Geriatr Res. 2008;2008 (February):1-11. doi:10.1155/2008/474868

24. Burmeister LA, Ganguli M, Dodge HH, Toczek T, Dekosky ST, Nebes RD. Hypothyroidism and cognition: preliminary evidence for a specific defect in memory. Thyroid. 2001;11(12):1177-1185. doi:10.1089/10507250152741037

25. Tefra M, Dessalegn Muluken BS. Cognitive impairment among type 2 diabetes mellitus patients in Ethiopia. Int J Med Appl Sci. 2013;2 (3):40-54.

26. Vertesi A, Lever JA, Molloy DW, et al. Standardized mini-mental state examination use and interpretation. Can Fam Physician. 2001;47(OCT):2018-2023.

27. Molloy DW. Standardised Mini-Mental State Examination (SMMSE)-Guidelines for administration and scoring instructions. Am J Psychiatry. 2014;14:102-105.

28. Mitchell AJ, Shukla D, Ajumal HA, Stubbs B, Tahir TA. The Mini-Mental State Examination as a diagnostic and screening test for delirium: systematic review and meta-analysis. Gen Hosp Psychiatry. 2014;36(6):627-633. doi:10.1016/j.genhosppsych.2014. 09.003

29. Cronbach LJ. Coefficient alpha and the internal structure of tests. Psychometrika. 1951;16(3):297-334. doi:10.1007/BF02310555

30. Nielsen-Bohlman L, Panzer AM, Kindig DA. Institute of Medicine (US) Committee on Health Literacy; Prescription to end confusion; 2004. Available from: https://www.ncbi.nlm.nih.gov/books/ NBK216033. Accessed March 16, 2021.

31. Puymirat J. Thyroid receptors in the rat brain. Prog Neurobiol. 1992;39:281-294. doi:10.1016/0301-0082(92)90019-B

32. Murman D. The Impact of Age on Cognition. Semin Hear. 2015;36 (03):111-121. doi:10.1055/s-0035-1555115

33. Sengupta P, Benjamin A, Singh Y, Grover A. Prevalence and correlates of cognitive impairment in a north Indian elderly population. WHO South-East Asia J Public Heal. 2014;3(2):135. doi:10.4103/ 2224-3151.206729

34. Bernal J. Thyroid hormones and brain development. Vitamins Horm. 2005;95-122.

35. Hogervorst E, Huppert F, Matthews FE, Brayne C. Thyroid function and cognitive decline in the MRC Cognitive Function and Ageing Study. Psychoneuroendocrinology. 2008;33(7):1013-1022. doi:10.10 16/j.psyneuen.2008.05.008 


\section{Publish your work in this journal}

Neuropsychiatric Disease and Treatment is an international, peerreviewed journal of clinical therapeutics and pharmacology focusing on concise rapid reporting of clinical or pre-clinical studies on a range of neuropsychiatric and neurological disorders. This journal is indexed on PubMed Central, the 'PsycINFO' database and CAS, and is the official journal of The International Neuropsychiatric Association (INA). The manuscript management system is completely online and includes a very quick and fair peer-review system, which is all easy to use. Visit http://www.dovepress.com/testimonials.php to read real quotes from published authors. 